Exp. Anim. 57(4), 401-405, 2008

- Note-

\title{
Factors Affecting Full-Term Development of Rat Oocytes Microinjected with Fresh or Cryopreserved Round Spermatids
}

\author{
Masumi HIRABAYASHI ${ }^{1,2)}$, Megumi KATO ${ }^{1)}$, and Shinichi $\mathrm{HOCHI}^{3)}$
}

\author{
${ }^{1)}$ National Institute for Physiological Sciences, Okazaki, Aichi 444-8787, \\ ${ }^{2)}$ The Graduate University for Advanced Studies, Okazaki, Aichi 444-8787, and \\ ${ }^{3)}$ Faculty of Textile Science and Technology, Shinshu University, Ueda, Nagano 386-8567, Japan
}

\begin{abstract}
Factors affecting full-term development of rat oocytes after round spermatid injection (ROSI) were examined using fresh and cryopreserved spermatids. Regardless of method (DC pulses or ionomycin) and timing (before or after ROSI) for oocyte activation, similar percentages of oocytes injected with cryopreserved spermatids developed to full-term (3.0-4.8\%). In contrast, no offspring were obtained when fresh spermatids were injected into $D C$-treated oocytes (0\%), but a higher proportion of oocytes first injected with fresh spermatids and then activated with ionomycin developed to full-term (6.1\%). In further experiment, oocytes activated with ionomycin were injected with frozen-thawed spermatids, and then treated with cycloheximide and/or trichostatin-A. Neither chemical had a beneficial effect on full-term development of ROSI oocytes (3.7-7.9\% vs $2.5 \%$ in control). In conclusion, regardless of timing of oocyte activation, activation treatment with ionomycin is required for full-term development of rat oocytes injected with fresh spermatids, and the higher potential of cryopreserved spermatids contributing to full-term development is notable when DC pulse is applied for oocyte activation.
\end{abstract}

Key words: cryopreservation, oocyte activation, round spermatid

Ogura et al. [12] were the first to obtain normal offspring after transfer of mouse oocytes electrically fused with round spermatids. Intracytoplasmic injection of round spermatids (ROSI) has also resulted in the birth of normal offspring in rabbits [16], mice [7], humans [17], rats [2], and hamsters [1]. Moreover, mouse offspring have been produced by injecting cryopreserved round spermatids [13]. Round spermatids are generally susceptible to metaphase-promoting factor (MPF) in the oocytes. Therefore to avoid premature chromosome condensation in response to the MPF, oocytes need to be activated prior to ROSI [11]. In rats, a ROSI protocol using cryopreserved round spermatids has been developed for the rescue of infertile transgenic rat lines [2], and the efficiency of offspring production has been improved by activating oocytes with a direct current (DC) pulse instead of $\mathrm{SrCl}_{2}$ prior to the ROSI [4]. However, this improved ROSI protocol is not valid for freshly

(Received 15 October 2007 / Accepted 1 January 2008)

Address corresponding: M. Hirabayashi, National Institute for Physiological Sciences, Okazaki, Aichi 444-8787, Japan

M. Hirabayashi and M. Kato contributed equally to this work. 
isolated round spermatids $[2,15]$. It was reported that developmental potential into blastocysts of bovine ROSI oocytes was improved by an activation regimen with both ionomycin and cycloheximide (CHX) [10]. Treatment of mouse round spermatids with trichostatin-A (TSA) inhibited global re-methylation of paternal genomes [8].

The objective of the present study was to examine the effect of different timings of two activation treatments on rat ROSI using fresh and cryopreserved spermatid cells in a $2 \times 2 \times 2$ factorial design experiment (Experiment 1 ). In addition, the ionomycin stimulus inducing single intracellular calcium increase was combined with CHX treatment to prevent re-accumulation of MPF, and/ or a TSA treatment to suppress histone de-acethylation (Experiment 2).

In Experiment 1, rat round spermatids were isolated as reported for mice by Ogura et al. [11]. Briefly, testes from mature Sprague-Dawley rats (Slc:SD; Japan SLC, Inc., Shizuoka, Japan) were placed in erythrocyte-lysing buffer (155 mM NH $\mathrm{m}_{4} \mathrm{Cl}, 10 \mathrm{mM} \mathrm{KHCO}, 2$ mM EDTA; $\mathrm{pH}$ 7.2), and tunica albuginea was removed. Seminiferous tubules were transferred into GL-PBS (Dulbecco's PBS supplemented with $5.6 \mathrm{mM}$ glucose, $5.4 \mathrm{mM}$ sodium lactate and $0.01 \%$ polyvinylpyrrolidone) at $4{ }^{\circ} \mathrm{C}$, cut into small pieces using a pair of fine scissors, and shaken gently to release spermatogenic cells into the medium. The cell suspension was filtered through a 40- $\mu \mathrm{m}$ nylon mesh and then centrifuged at $200 \times g$ for 5 $\min$ at $4^{\circ} \mathrm{C}$. Cells were resuspended in GL-PBS containing $0.2 \mathrm{mg} / \mathrm{ml}$ pronase $\mathrm{E}$ (Sigma-Aldrich, St. Louis, $\mathrm{MO}$, USA). After centrifugation at $400 \times g$ for $5 \mathrm{~min}$ at $4{ }^{\circ} \mathrm{C}$, elongating spermatids and testicular spermatozoa which agglutinated into sticky masses were discarded from the suspension. The remaining suspension, expected to be rich in round spermatids, was washed twice with GLPBS by centrifugation at $200 \times g$ for 5 min each at $4^{\circ} \mathrm{C}$. The spermatids were either used when freshly isolated, or used after cryopreservation in GL-PBS supplemented with 7.5\% glycerol (v/v; Wako Pure Chemical Industries, Osaka, Japan) and 7.5\% fetal bovine serum (v/v; FBS; Equitech Bio, Ingram, TX, USA).

Oocyte recovery and ROSI were performed as reported previously by Hirabayashi et al. [2]. Briefly, Slc:SD of 4- to 6-week-old female rats were superovu- lated by intraperitoneal injections of $300 \mathrm{IU} / \mathrm{kg}$ equine chorionic gonadotrophin (eCG; Nippon Zenyaku Kogyo, Fukushima, Japan) and 300 IU/kg human chorionic gonadotrophin (hCG; Sankyo Yell Yakuhin, Tokyo, Japan) at an interval of $48 \mathrm{~h}$. Oocytes were collected from the oviductal ampullae 14 to $17 \mathrm{~h}$ after the hCG injection, and were freed from cumulus cells by 5 to 10 min treatment with $0.1 \%$ hyaluronidase (w/v; Sigma-Aldrich) dissolved in $22 \mathrm{mM}$ Hepes-buffered $\mathrm{mR} 1 \mathrm{ECM}$ medium [14]. Ten to fifteen oocytes were placed in a $10-\mu 1$ drop of the Hepes-buffered mR1ECM medium, and $1 \mu \mathrm{l}$ of fresh or frozen-thawed spermatid suspension ( 2 to $5 \times$ $10^{5}$ cells $/ \mathrm{ml}$ ) was placed in a $10-\mu 1$ drop of the Hepesbuffered mR1ECM medium supplemented with $8 \%$ polyvinylpyrrolidone (PVP, $360 \mathrm{kDa}$; ICN Pharmaceuticals, Inc., Costa Mesa, CA, USA). Individual round spermatids were picked up with a blunt-ended injection pipette having an inner diameter of 5 to $6 \mu \mathrm{m}$, and the spermatid membrane was broken by shear stress with repeated pipetting. Microinjection of spermatids into oocytes was performed at ambient temperature using a piezo impact driving unit (PMM-150FU; Prime Tech, Ibaraki, Japan) with a pulse controller (PMASCT150; Prime Tech).

One of two different regimens (DC pulses or ionomycin) was applied to activate oocytes $40 \mathrm{~min}$ before or 10 min after ROSI. For DC pluse treatment oocytes were transferred into DC activation solution ( $0.3 \mathrm{M}$ mannitol, $0.1 \mathrm{mM} \mathrm{CaCl}_{2}, 0.1 \mathrm{mM} \mathrm{MgSO}_{4}$, and $0.1 \%$ fatty acid-free bovine serum albumin; Sigma-Aldrich) and then placed between the electorode gap (width $0.5 \mathrm{~mm}$, depth 2.0 $\mathrm{mm}$ ) of a circular electrofusion chamber (FTC-01; Shimadzu Scientific Instruments, Kyoto, Japan) containing $20 \mu 1$ of DC activation solution. A DC pulse at $50 \mathrm{~V} /$ $\mathrm{mm}$ was applied twice to the oocytes for a duration of $99 \mu \mathrm{sec}$ each at an interval of $1 \mathrm{sec}$ using an electro-cell fusion generator (LF101; Nepa Gene, Chiba, Japan). The oocytes were then washed twice with mR1ECM. For ionomycin treatment oocytes were incubated for 5 min in mR1ECM medium containing $5 \mu \mathrm{M}$ ionomycin (Sigma-Aldrich) at ambient temperature and washed twice with mR1ECM medium. ROSI oocytes were cultured in $60 \mu 1$ microdrops of $\mathrm{mR} 1 \mathrm{ECM}$ medium at $37^{\circ} \mathrm{C}$ under mineral oil in $5 \% \mathrm{CO}_{2}$ in air. All non-degenerating one-cell oocytes and evenly cleaved two-cell oocytes 
Table 1. Full-term development of rat oocytes microinseminated with fresh or cryopreserved round spermatids

\begin{tabular}{|c|c|c|c|c|c|c|c|c|}
\hline \multirow{2}{*}{$\begin{array}{c}\text { Source of } \\
\text { spermatids }\end{array}$} & \multirow{2}{*}{$\begin{array}{l}\text { Activation } \\
\text { regimen }\end{array}$} & \multirow{2}{*}{$\begin{array}{c}\text { Activation } \\
\text { timing }\end{array}$} & \multirow{2}{*}{$\begin{array}{l}\text { No. of } \\
\text { replicates }\end{array}$} & \multicolumn{3}{|c|}{ No. $(\%)$ of oocytes } & \multicolumn{2}{|c|}{ No. (\%) of zygotes } \\
\hline & & & & Examined & $2 \mathrm{PN}+\mathrm{pb}^{*}$ & Cleaved* & Transferred & Developed to term** \\
\hline \multirow[t]{4}{*}{ Fresh } & DC pulse & Pre-ROSI & 4 & 157 & $13(8.3)$ & $24(15.3)$ & 131 & $0(0)$ \\
\hline & & Post-ROSI & 4 & 109 & $7(6.4)$ & $20(18.3)$ & 100 & $3(3.0)$ \\
\hline & Ionomycin & Pre-ROSI & 4 & 130 & $23(17.7)$ & $20(15.4)$ & 111 & $4(3.6)$ \\
\hline & & Post-ROSI & 4 & 150 & $19(12.7)$ & $36(24.0)$ & 131 & $8(6.1)$ \\
\hline \multirow[t]{4}{*}{ Cryopreserved } & DC pulse & Pre-ROSI & 4 & 131 & $36(27.5)$ & $54(41.2)$ & 105 & $5(4.8)$ \\
\hline & & Post-ROSI & 4 & 128 & $24(18.8)$ & $43(33.6)$ & 99 & $3(3.0)$ \\
\hline & Ionomycin & Pre-ROSI & 4 & 134 & $36(26.9)$ & $55(41.0)$ & 110 & $4(3.6)$ \\
\hline & & Post-ROSI & 4 & 145 & $40(27.6)$ & $49(33.8)$ & 118 & $4(3.4)$ \\
\hline
\end{tabular}

* Percentages for $2 \mathrm{PN}+\mathrm{pb}$ (two pronuclei+second polar body) and first cleavage were calculated from the number of oocytes examined. Significantly different between "Fresh" and "Cryopreserved" ( $P<0.01$; three-way ANOVA). **Percentage for full-term development was calculated from the number of zygotes transferred. Significantly different between "Fresh" and "Cryopreserved" in case of DC pulse $(P<0.05$; three-way ANOVA) and between "DC pulse" and "Ionomycin" in case of Fresh spermatids $(P<0.05$; three-way ANOVA).

at 23 to $25 \mathrm{~h}$ after ROSI were transferred into the oviductal ampullae of recipient Slc:Wistar Hannover/Rcc rats (13 to 22 -weeks-old) that had previously been mated with vasectomized male rats. Embryo transfer was performed on the day that a vaginal plug was detected (defined to as Day 1). On Day 21, offspring were delivered from the recipients by Caesarean section. Experiments were repeated three or four times. Proportional data of each replicate were arcsin-transformed and subjected to three-way ANOVA (Experiment 1) using JavaScript-STAR program (version 4.1.1j: http://www. kisnet.or.jp/nappa/software/star/) or one-way ANOVA and Scheffe's F-test (Experiment 2) using StatView software (Abacus Concepts, Berkeley, CA).

As shown in Table 1, rat oocytes after microinjection of both fresh and cryopreserved round spermatids developed to full-term. Three-way ANOVA indicated that, regardless of method and timing of oocyte activation, both proportions of oocytes exhibiting formation of two pronuclei with polar body extrusion $(2 \mathrm{PN}+\mathrm{pb})$ and cleaving were significantly different between fresh and cryopreserved spermatids $(\mathrm{F}=11.18, P<0.01$ and $\mathrm{F}=14.79$, $P<0.01$, respectively). Oocytes injected with fresh spermatids appeared to have delayed pronucleus formation and first cleavage when compared with those injected with cryopreserved spermatids (data not shown). However, the time interval between ROSI and embryo transfer ( 23 to $25 \mathrm{~h}$ ) was fixed throughout the experiments because a longer culture period in the same mR1ECM medium is not suitable for the early cleaving oocytes. It is well-known that culture medium with a lower osmolality is required for further development of rat embryos beyond the two-cell stage [5,9]. Further investigation taking into consideration the poor correlation of cleavage rate with offspring rate is necessary. As to the percentage of transferred oocytes developing to full-term, there was a significant interaction between the source of spermatids (fresh or cryopreserved) and method of activation (DC pulse or ionomycin). When DC pulse was applied for oocyte activation, a higher percentage of oocytes injected with cryopreserved spermatids developed to full-term $(\mathrm{F}=6.12, P<0.05)$. Furthermore, within the groups of oocytes injected with fresh spermatids, oocyte activation with ionomycin resulted in higher full-term development rates than with the DC pulse treatment, regardless of activation timing $(\mathrm{F}=4.86, P<0.05)$. The action of DC pulse and ionomycin as triggers for oocyte activation needs to be re-evaluated by monitoring the kinetics of both the intracellular calcium level and decondensation of the spermatid nucleus.

In Experiment 2, rat oocytes were activated by $5 \mathrm{~min}$ treatment with $5 \mu \mathrm{M}$ ionomycin $40 \mathrm{~min}$ before ROSI using cryopreserved spermatids, and then the ROSI oocytes were treated for $4 \mathrm{~h}$ with $5 \mu \mathrm{g} / \mathrm{ml} \mathrm{CHX} \mathrm{(Sigma-}$ Aldrich) and/or for $10 \mathrm{~h}$ with $5 \mathrm{nM} \mathrm{TSA}$ (Sigma-Aldrich). In this experiment, spermatid suspensions were frozen using solution of Cell-Banker 1 (BLC-1; Juji Field Inc., Tokyo, Japan). The method of spermatid cryopreserva- 
Table 2. Effect of trichostatin-A and cycloheximide treatments on normal fertilization and full-term development of spermatid-injected rat oocytes

\begin{tabular}{lccccccc}
\hline & \multirow{2}{*}{$\begin{array}{c}\text { No. of } \\
\text { Groups }\end{array}$} & \multicolumn{3}{c}{ No. (\%) of oocytes } & & \multicolumn{2}{c}{ No. (\%) of zygotes } \\
\cline { 3 - 4 } Control & 4 & 152 & $30(19.7)^{\mathrm{a}}$ & $13(8.6)$ & & 118 & $3(2.5)$ \\
TSA & 4 & 144 & $30(20.8)^{\mathrm{a}}$ & $10(6.9)$ & & 110 & $6(5.5)$ \\
CHX & 3 & 113 & $93(82.3)^{\mathrm{b}}$ & $23(20.4)$ & & 89 & $7(7.9)$ \\
TSA/CHX & 3 & 109 & $82(75.2)^{\mathrm{b}}$ & $21(19.3)$ & & 81 & $3(3.7)$ \\
\hline
\end{tabular}

* Percentages for $2 \mathrm{PN}+\mathrm{pb}$ (two pronuclei+second polar body) and first cleavage were calculated from the number of oocytes examined. **Percentage for full-term development was calculated from the number of zygotes transferred. TSA; Trichostatin-A, CHX: Cycloheximide, PN: Pronuclei, pb: Polar body. Oocytes were first activated with ionomycin, and then injected with frozen-thawed spermatids. ${ }^{a, b}$ Different superscripts denote significant difference $(P<0.05$; one-way ANOVA).

tion (glycerol/FBS-based solution vs BLC-1 solution) did not affect the full-term development of ROSI oocytes (3.6\% in Table 1 vs $2.5 \%$ in Table 2). Significantly higher percentages of ROSI oocytes exhibited a sign of normal fertilization $(2 \mathrm{PN}+\mathrm{pb})$ when the ROSI oocytes were treated with CHX (75.2-82.3\% vs $19.7-20.8 \%$; $P<0.05)$, as shown in Table 2. However, no positive effect of the CHX and/or TSA treatments on full-term development of ROSI oocytes was detected, as the offspring rates were $2.5,5.5,7.9$, and $3.7 \%$ in the control, TSA, CHX, and TSA/CHX groups, respectively. It remains for further research to examine if the concentration and treatment duration of the TSA/CHX determined in previous studies $[8,10]$ are optimal for rat ROSI oocytes.

Microinsemination of spermatogenic cells differentiated from germ stem cells is an alternative approach for producing gene-targeted rats, because neither embryonic stem cell line nor somatic cell nuclear transfer protocol has been established in the rat. From this point of view, maximum use of selected spermatid cells for offspring production must be important. Since one rat offspring was produced after transfer of 206 oocytes injected with freshly isolated round spermatids [2], no more offspring have been obtained using the same regimen in our laboratory. Recently, our attempt at ROSI using fresh rat spermatids that differentiated after transplantation of spermatogonial stem cells into immunodeficient mice also resulted in failure to produce offspring, while ROSI after cryopreservation of the same spermatids gave birth to normal rat offspring [15]. The beneficial effect of spermatid cryopreservation on microin- semination in rats may be due to facilitation of nuclear envelope breakdown of spermatid cells, forwarding the paternal nuclear materials to contribute to the normal post-fertilization events. On the other hand, freshly isolated spermatids with a highly integrated nuclear envelope might need to be exposed for a longer period to the relatively high MPF activity in the oocytes, even though rat oocytes activate spontaneously once they are released from oviductal ampullae $[3,6,18]$. Our results suggest that both overall integrity of spermatids (influenced by cryopreservation) and MPF activity of ooplasm (influenced by method and timing of oocyte activation) affect the nuclear envelope breakdown of injected spermatid cells, which is followed by decondensation of paternal chromatin and subsequent formation of the male pronucleus. Regarding the low offspring rate after transfer of rat ROSI oocytes (up to 7.9\% in the present study), more effort should be made to target the current mouse ROSI offspring rate of $>30 \%$.

In conclusion, regardless of timing of oocyte activation, activation treatment with ionomycin is required for full-term development of rat oocytes injected with fresh spermatids, and the higher potential of cryopreserved spermatids contributing to full-term development is notable when a DC pulse is applied for oocyte activation. We suggest that nuclear envelope breakdown of injected spermatid cells is closely associated with the interaction of overall integrity of spermatids and MPF activity of ooplasm. Normal embryogenesis of ROSI oocytes may be triggered only when the physiological change of paternal chromatin occurs at optimal timing. 


\section{Acknowledgment(s)}

This work was supported in part by Grant-in-Aids for the Scientific Research on Priority Areas (No. 15082211) from the Ministry of Education, Culture, Sports, Science and Technology of Japan, and for the Scientific Research (No. 17658124, No. 19300150) from the Japan Society for the Promotion of Science.

\section{References}

1. Haigo, K., Yamauchi, Y., Yazama, F., Yanagimachi, R., and Horiuchi, T. 2004. Biol. Reprod. 71: 194-198.

2. Hirabayashi, M., Kato, M., Aoto, T., Ueda, M., and Hochi, S. 2002. Mol. Reprod. Dev. 62: 295-299.

3. Ito, J., Hirabayashi, M., Kato, M., Takeuchi, A., Ito, M., Shimada, M., and Hochi, S. 2005. Reproduction 129: 171-180.

4. Kato, M., Ishikawa, A., Hochi, S., and Hirabayashi, M. 2004. Contemp. Top. Lab. Anim. Sci. 43: 13-15.

5. Kato, M., Ishikawa, A., Hochi, S., and Hirabayashi, M. 2004. J. Reprod. Dev. 50: 191-195.

6. Keefer, C.L. and Schuetz, A.W. 1982. J. Exptl. Zool. 224: 371-377.

7. Kimura, Y. and Yanagimachi, R. 1995. Development 121:
2397-2405.

8. Kishigami, S., Van Thuan, N., Hikichi, T., Ohta, H., Wakayama, S., Mizutani, E., and Wakayama, T. 2006. Dev. Biol. 289: 195-205.

9. Miyoshi, K., Kono, T., and Niwa, K. 1997. Biol. Reprod. 56: $180-185$.

10. Ock, S.A., Kwack, D.O., Lee, S.L., Cho, S.R., Jeon, B.G., Mohana kumar, B., Choe, S.Y., and Rho, G.J. 2006. Theriogenology 65: 1242-1253.

11. Ogura, A. and Yanagimachi, R. 1993. Biol. Reprod. 48: 219-225.

12. Ogura, A., Matsuda, J., and Yanagimachi, R. 1994. Proc. Natl. Acad. Sci. U.S.A. 91: 7460-7462.

13. Ogura, A., Matsuda, J., Asano, T., Suzuki, O., and Yanagimachi, R. 1996. J. Assist. Reprod. Genet. 13: 431-434.

14. Oh, S.H., Miyoshi, K., and Funahashi, H. 1998. Biol. Reprod. 59: 884-889.

15. Shinohara, T., Kato, M., Takehashi, M., Lee, J.Y., Chuma, S., Nakatsuji, N., Kanatsu-Shinohara, M., and Hirabayashi, M. 2006. Proc. Natl. Acad. Sci. U.S.A. 103: 13624-13628.

16. Sofikitis, N.V., Miyagawa, I., Agapitos, E., Pasyianos, P., Toda, T., Hellstrom, W.J., and Kawamura, H. 1994. J. Assist. Reprod. Genet. 11: 335-341.

17. Tesarik, J., Mendoza, C., and Tesarik, J. 1995. N. Engl. J. Med. 333: 525.

18. Zernicka-Goetz, M. 1991. Mol. Reprod.Dev. 28: 169-176. 